\title{
El impacto de la pobreza y la violencia en la salud y los derechos reproductivos de las mujeres en El Salvador
}

\author{
The impact of poverty and violence against \\ women's reproductive health and rights \\ in El Salvador
}

J. Sebastián Rodríguez Alarcón 1

Maria Fernanda Perico 2

$10.1590 / 0102-311 \times 00039119$

\section{Introducción}

El Salvador cuenta con una de las legislaciones más restrictivas en el mundo en materia de aborto 1. Hasta 1998, El Salvador permitía el aborto en tres casos específicos, incluyendo los casos en los que fuera necesario proteger la vida de la mujer, cuando el embarazo fuera producto de un delito, así como en los casos de malformación fetal incompatible con la vida extrauterina 2 . Sin embargo, en 1998 el Código Penal fue reformado, penalizando el aborto en todos los casos, al igual que criminalizando a los empleados públicos de cualquier autoridad que no reporten su comisión, incluyendo aquellos funcionarios que trabajan en hospitales y clínicas. El cambio en la ley desde 1998 generó como consecuencia paralela que profesionales médicos, en contravía del derecho a la confidencialidad de los pacientes, reporten ante las autoridades a las mujeres que acceden a los servicios de emergencia por causas de salud reproductiva, tales como, por ejemplo, emergencias obstétricas, bajo la sospecha de que han tenido abortos inducidos 3 .

Tras los cambios legislativos de 1998, El Salvador también modificó su Constitución Politica en 1999, reconociendo al embrión como un ser humano desde "el momento de la concepción" 4. Así, una persona que realiza o se autoinduce un aborto, incluso antes de la etapa fetal, puede ser procesada por el delito de homicidio que conlleva una pena de prisión de hasta 50 años de cárcel 1 .

Las consecuencias de la aplicación de la ley en El Salvador han generado como resultado que mujeres que enfrentan complicaciones en sus embarazos, incluyendo emergencias obstétricas, prefieren no buscar asistencia médica por el temor de ser perseguidas, investigadas y encarceladas bajo la sospecha de haber tenido abortos. En los casos en que las mujeres efectivamente acceden al aborto, una vez reportadas a las autoridades, corren el riesgo de enfrentarse a las fallas que permean el sistema judicial, sufriendo graves violaciones en el debido proceso durante la etapa judicial, donde en varios casos ni siquiera cuentan con la adecuada representación legal 5.

Adicionalmente, otros factores contribuyen a que se generen barreras en el acceso a servicios de salud reproductiva de las mujeres en El Salvador. La desigualdad socioeconómica, por ejemplo, es la principal causa de la inequidad de género e impide el acceso a los servicios de salud por parte de las mujeres, en particular mujeres rurales y de escasos recursos. Asimismo, los factores socioculturales relacionados con estereotipos de género y patrones marcados de violencia contra las mujeres, sumado a la causa raíz del problema, que obedece a la legislación restrictiva que impide el acceso al aborto en
1 Center for Reproductive Rights, New York, U.S.A. 2 Center for Reproductive Rights, Bogotá, Colombia.

\section{Correspondencia}

M. F. Perico

mperico@reprorights.org 
todos los casos, también contribuye de manera significativa al ambiente de discriminación estructural contras las mujeres.

Es a partir de este contexto legal que se desata un ambiente de estigma y discriminación estructural en contra de las mujeres en el país ${ }^{6}$. Aquellas personas que acceden a los servicios de salud reproductiva o expresan su posición a favor de este, pueden estar sujetas a abuso, agresión, violencia política, persecución y hostigamiento 7 .

\section{La pobreza y el desarrollo como factores clave que contribuyen a la discriminación estructural en contra de las mujeres en el ámbito de la salud reproductiva en El Salvador}

Con una población cercana los 6.1 millones de personas (Banco Mundial. Indicadores del desarrollo mundial, El Salvador: Población total; 2017. http://data.worldbank.org/country/el-salvador, accedido el 04/Mar/2019), El Salvador es un país de ingreso medio-bajo con un crecimiento del Producto Interno Bruto (PIB) del 2,3\% en 2017 8. Comparativamente en la región, el país enfrenta bajos niveles de crecimiento persistentes. Entre 2010 y 2016, el crecimiento del PIB real promedio fue del 2,6\%, convirtiendo a El Salvador en uno de los países con el crecimiento económico más bajo en la región de América Central 8.

En las últimas décadas, pese a que la población de El Salvador ha venido trasladándose del campo a la ciudad cada vez más (con un 67\% de los habitantes del país viviendo en zonas urbanas 9), la pobreza en el país es altamente generalizada, con cerca del 32\% de la población viviendo por debajo de la línea nacional de pobreza. La pobreza es aún más alta en las áreas rurales donde aproximadamente el 38\% de la población vive en pobreza absoluta, es decir, por debajo de la línea de pobreza nacional 10.

La población adolescente representa cerca del 34\% del total de la población en El Salvador 11, que, a su vez, resulta ser la población más pobre del país 12. Atados a los altos niveles de pobreza, los adolescentes y jóvenes se enfrentan a varios desafíos interrelacionados de salud reproductiva para satisfacer sus necesidades y potencial. Las mujeres y las niñas en El Salvador son el sector poblacional más afectado por la pobreza, registrando los niveles más bajos de educación, las tasas más altas de deserción escolar, y aproximadamente el doble de la tasa de analfabetismo que los hombres. Así, las mujeres salvadoreñas se ven enfrentadas a un perverso ciclo de pobreza, ya que, al no contar con acceso efectivo y de calidad a la educación, y, por ende, a las habilidades técnicas y profesionales suficientes, también se enfrentan como resultado a una menor participación en la fuerza laboral formal $\mathrm{y}$, en consecuencia, registran de manera significativa menores ingresos económicos en el transcurso de sus vidas, lo que limita la igualdad de oportunidades entre hombres y mujeres en la sociedad 13 .

Adicionalmente a las barreras económicas, El Salvador es uno de los países más violentos en el mundo. Gran parte de la violencia en el país es impulsada por grandes pandillas y redes de tráfico de drogas, que resultan en una de las tasas de homicidio, hostigamiento y amenazas más altas, que se enmarcan en un contexto persistente y generalizado de violencia que afecta la mayoría de la población en El Salvador. La restricción de movimiento de las personas dentro del país, producto en su mayoría de la violencia generalizada, ha llevado consigo a un desplazamiento interno significativo de la población. Por ejemplo, una encuesta encontró que en el 2014 cerca de 300.000 de los residentes del país, un número igual a toda la población de San Salvador, fueron desplazados por causas de la violencia y las amenazas 14 .

El bajo crecimiento económico y la pobreza, sumado al contexto generalizado de violencia, tiene un impacto en el acceso a la salud de las personas en el país. Pese a que en los últimos años ha habido un aumento en el acceso a los servicios de salud por parte de los sectores de la población más vulnerables, aún existen grandes desafíos en materia de acceso a los servicios de salud 8 .

Conforme a la Organización Mundial de la Salud (OMS), existen importantes desigualdades en el financiamiento de la atención de salud entre los diferentes subsistemas de salud y el servicio nacional en El Salvador, lo que ha llevado a una persistente carencia de fondos suficientes para financiar el sistema, no solo en materia de salud reproductiva, sino en general 15. Lo anterior ha resultado en que El Salvador sea uno de los pocos países de América Latina considerados en crisis con respecto a recursos humanos para la salud 15,16. El alto costo que involucra la atención de salud, a su vez, ha generado un 
menor uso de los servicios de salud en El Salvador, lo que ha representado una barrera para el acceso a los servicios de salud por parte de toda la población 15 .

En materia de estereotipos culturales de género, El Salvador también presenta una cultura sexista arraigada que se manifiesta en varios aspectos de la vida de las mujeres, tales como en sus relaciones íntimas que desencadenan en altos niveles de violencia intrafamiliar 17. Cerca de la mitad de las mujeres salvadoreñas que han estado en una relación íntima, reportan haber experimentado violencia al menos una vez por parte de su pareja 13. Adicionalmente, el país reporta un número creciente de violaciones sexuales que afecta particularmente a las mujeres 13.

La violencia basada en el género tiene efectos significativos en la salud reproductiva de las mujeres, especialmente cuando la violencia está directamente relacionada con la salud reproductiva. Esto se manifiesta de manera directa, por ejemplo, cuando se obliga a una persona a usar o no usar anticonceptivos o, cuando se obliga a una persona para que aborte o no; o de manera indirecta, cuando una persona agrede de manera física o mental a otra para evitar que esta ejerza su plena libertad reproductiva 11.

Así, el sexismo arraigado, la falta de oportunidad y dependencia socioeconómica, sumado al contexto de violencia generalizada y de género, genera un ambiente que limita el ejercicio de los derechos reproductivos de las mujeres en igualdad de condiciones que los hombres. Por ejemplo, las mujeres ocasionalmente no pueden pagar los costos de transporte necesarios para buscar atención médica cuando lo necesitan; en otras circunstancias dependen del apoyo económico de los hombres para consultar a un profesional de la salud 18 .

\section{Las consecuencias de la restricción legal absoluta del aborto en El Salvador}

\section{Consecuencias en la salud física y mental de las mujeres}

Con base en el contexto de pobreza y violencia generalizada em El Salvador. Estos factores tienen un impacto en el acceso a los servicios de salud física y mental de la mujeres, particularmente en el ámbito de la salud reproductiva. Cuando el aborto es realizado en un ambiente adecuado con supervisión y asistencia médica técnica y calificada, es un procedimiento seguro 19. Sin embargo, la OMS ha notado que "las mujeres, incluso las adolescentes con embarazos no deseados, a menudo recurren al aborto inseguro cuando no pueden acceder al aborto seguro" 20. El aborto inseguro se produce "cuando una persona carente de la capacitación necesaria pone fin a un embarazo, o se hace en un entorno que no cumple las normas médicas mínimas, o cuando se combinan ambas circunstancias" 20. Esto ocurre especialmente en los países en los que el aborto está completamente prohibido o solo está permitido en circunstancias muy limitadas. En estos lugares solo uno de cada cuatro abortos es seguro $21 \mathrm{y}$, adicionalmente, se producen "la mayoría de las muertes y morbilidad por abortos inseguros" 19.

El aborto inseguro se cobra la vida de 47.000 mujeres cada año 22, con casi todas estas muertes ocurriendo en regiones de bajo o mediano ingreso (Banco Mundial. https://blogs.worldbank.org/es/ opendata/nueva-clasificacion-de-los-paises-segun-el-nivel-de-ingreso-para-el-periodo-2018-19). El aborto inseguro puede llevar a una variedad de daños que afectan la calidad de vida y el bienestar de las mujeres, lo que incluye la posibilidad de complicaciones físicas, tales como: abortos incompletos, infecciones, perforaciones uterinas, enfermedades inflamatorias pélvicas, hemorragias $\mathrm{u}$ otras lesiones internas que pueden causar la muerte de la mujer o generar lesiones permanentes o infertilidad 13,20,23.

En materia de salud mental, debido al estigma asociado con el procedimiento y al temor real de ser reportada ante las autoridades, esto puede también generar un mayor riesgo de complicaciones de salud mental al largo plazo, o incluso la muerte 24 . Por ejemplo, en los casos de embarazos no planificados, la restricción total también pone a las mujeres en un riesgo significativo, ya que se les obliga a llevarlos a término con posibles consecuencias graves para su salud y la de sus bebés.

En el caso específico de El Salvador, donde existe un patrón de criminalización a las mujeres que acceden a servicios de salud reproductiva, el solo hecho de estar embarazada, y saber que hay posibilidades de tener a lo largo del embarazo una emergencia obstétrica, puede significar una afectación en la salud mental de la mujer embarazada, por el temor y angustia que genera la posibilidad de ser procesada penalmente sólo por tener ese tipo de emergencia 25. 
Adicionalmente, cuando el embarazo es adolescente y no fue planificado puede ser asociado con emociones de vergüenza y estigma, el cual puede provocar rechazo por parte de las familias y las comunidades, y secuelas en la salud mental, incluyendo síntomas de ansiedad, depresión, y un mayor riesgo de suicidio 26 . Desde 2011, al menos 42 niñas o adolescentes embarazadas se han suicidado en El Salvador 27. Estas muertes se han asociado con casos de menores embarazadas que terminan sus vidas ante la falta de opciones para embarazos no deseados como resultado de una violación sexual, lo que conlleva a la discriminación y el estigma social relacionado con los estereotipos que recaen sobre el rol reproductivo de la mujer en la sociedad 27.

\section{El caso de las 9 mujeres contra El Salvador ante el Sistema Interamericano de Protección de los Derechos Humanos}

La prohibición absoluta del aborto ha llevado a que en El Salvador un número alarmante de mujeres 28,29, mayoritariamente mujeres jóvenes, rurales, con bajo nivel de escolarización y de escasos o nulos ingresos económicos 30, sean acusadas, judicializadas, condenadas y encarceladas por el hecho de sufrir emergencias obstétricas 31 .

En el 2015, grupos de derechos humanos denunció la responsabilidad internacional del Estado salvadoreño ante la Comisión Interamericana de Derechos Humanos (CIDH) en perjuicio de nueve mujeres que tuvieron emergencias obstétricas y fueron criminalizadas, debido a la prohibición total del aborto en el país. Cada uno de estos nueve casos refleja las múltiples barreras que el Estado impone para el acceso y garantía del derecho a la salud reproductiva de las mujeres salvadoreñas, así como las fallas estructurales del sistema judicial que desencadenaron en graves violaciones en el debido proceso y las graves condiciones de reclusión en las cárceles 31 .

En la mayoría de los casos, las mujeres que acudieron al sistema de salud durante el embarazo no recibieron una atención aceptable y accesible, ya sea porque recibieron información incompleta y sesgada sobre el diagnóstico prenatal (en los casos en que los fetos eran incompatibles con la vida extrauterina) (Naciones Unidas. Consejo Económico y Social. https://www.acnur.org/fileadmin/ Documentos/BDL/2001/1451.pdf) o porque no fueron informadas de que su embarazo implicaba un peligro para su vida o su salud 32 . Adicionalmente, cuando algunas de ellas acudieron a las instituciones de salud en situación de gravedad por las emergencias obstétricas sufridas, en lugar de proveerles los servicios médicos que requerían ${ }^{32}$, el personal médico las denunció ante las autoridades de policía por un delito que no cometieron ${ }^{33}$. Lo anterior, no solo es contrario al deber de secreto profesional ${ }^{1}$, sino al derecho a la confidencialidad de las pacientes, protegido bajo la ley penal salvadoreña ${ }^{3}$.

Varias de las nueve mujeres fueron esposadas cuando todavía se encontraban recibiendo tratamiento médico, o fueron maltratadas verbalmente antes y durante la detención. Asimismo, las nueve mujeres fueron privadas de libertad en condiciones extremas de hacinamiento, insalubridad y sin acceso a servicios de salud que cumplan con los estándares de disponibilidad y calidad (Naciones Unidas. Consejo Económico y Social. https://www.acnur.org/fileadmin/Documentos/BDL/2001/1451.pdf).

El Salvador tiene las cifras más altas de encarcelación de su historia 34,35 , con un hacinamiento carcelario que alcanza el 230\% 36. Según cifras generales del sistema penitenciario, en los años 2009, 2015 y 2018, la población privada de libertad fue, respectivamente, de 21.032, 31.148 y 39.274 internos 36. Así, se observan incrementos alarmantes en el número de personas privadas de la libertad, arrojando una tasa de 614 personas presas por cada 100.000 habitantes 34,37. En mayo 2016 la Sala de lo Constitucional de la Corte Suprema de Justicia se manifestó al respecto y declaró el estado de cosas inconstitucional ante las condiciones de hacinamiento de los centros de reclusión en El Salvador (https://sv.vlex.com/vid/569851122, accedido el 27/May/2016).

El Centro de Readaptación para Mujeres "Ilopango" 38, en donde estuvieron privadas de la libertad algunas de las nueve mujeres, reporta uno de los mayores niveles de hacinamiento del país (367\%) 34 . En los años 2014, 2017 y 2018, respectivamente, albergaba un total de 2.073, 2.444 y 2.462 privadas de libertad 34 , lo que refleja también un continuo incremento de su población.

El hacinamiento genera impactos negativos en la salubridad del sistema penitenciario. En febrero de 2018 la Relatora Especial sobre las ejecuciones extrajudiciales, sumarias o arbitrarias de las Naciones Unidas expresó su preocupación al respecto, ya que las condiciones extremas de hacinamiento carcelario en El Salvador provocan la proliferación de enfermedades, como los recientes brotes de tubercu- 
losis registrados en los centros penales del país (Callamard A. Declaración final de misión en El Salvador. https://www.ohchr.org/SP/NewsEvents/Pages/DisplayNews.aspx?NewsID=22634\&LangID=S, accedido el 4/Mar/2019). Aunado a ello, los techos, sistemas de drenaje y tuberías de aguas negras de los centros penitenciarios están a punto de colapsar y se inundan en invierno 39 , poniendo así a las personas privadas de la libertad en múltiples formas de vulnerabilidad y violación de sus derechos.

A julio de 2015 solo había un profesional de la salud por cada cinco mil personas privadas de libertad, las clínicas de los centros no contaban con personal suficiente, equipo, ni los medicamentos necesarios para atender emergencias o tratar enfermedades graves y no disponían de custodios, ambulancias, o vehículos suficientes para trasladar a los reclusos que se encuentran enfermos a las consultas externas ${ }^{34}$. Así, en Centro Penitenciario para Mujeres "Granja Izalco" (en donde se encuentran las mujeres privadas de la libertad en fase de confianza $34 \mathrm{o}$ aquellas en fase ordinaria que tienen hijos menores de 5 años ${ }^{34}$ ) se ha reportado que ni las mujeres, ni los niños y niñas reciben atención médica, alimentación, agua y cuidados adecuados (http://elintercamb.io/ninospresos/elsalvador/, accedido el 05/Mar/2019).

De acuerdo con los pronunciamientos del relator especial sobre la tortura y outros tratos o penas crueles, inhumanos o degradantes, estas actuaciones son constitutivas de tratos crueles, inhumanos o degradantes 40 (Méndez JE. Informe del relator especial sobre la tortura y otros tratos o penas crueles, inhumanos o degradantes. https://www.ohchr.org/SP/Issues/Torture/SRTorture/Pages/SRTorture Index.aspx, accedido el 04/Mar/2019).

El caso de las nueve mujeres demuestra las consecuencias que la prohibición absoluta del aborto en El Salvador tiene para la garantía del derecho a la salud de las mujeres en el país. En efecto, la legislación salvadoreña no solo imposibilita el acceso a servicios esenciales de salud reproductiva, como la atención médica adecuada para mujeres que sufren emergencias obstétricas, sino que expone cómo el Estado, al privar injustamente de la libertad a las mujeres, continúa fallando en proveer la atención de salud que requieren mientras están en el sistema penitenciario, y se espera que la Comisión Interamericana de Derechos Humanos se pronuncie en esse sentido.

\section{Recomendaciones hechas por cuerpos internacionales de derechos humanos para reformar la ley restrictiva que restringe los derechos reproductivos en El Salvador}

Debido a que la ley nacional restringe el reconocimiento de los derechos reproductivos de las mujeres en el país, es necesario hacer referencia al marco legal internacional, a fines de remitir al reconocimiento que diversos cuerpos internacionales de derechos humanos han hecho en la materia, incluyendo la publicación de recomendaciones específicas al país, con el fin de que este reforme su legislación restrictiva en materia de derechos reproductivos.

El Salvador forma parte de varios instrumentos internacionales de derechos humanos que protegen los derechos fundamentales de las mujeres y el derecho al más alto nivel posible de salud, entre otros derechos socioeconómicos. Bajo el derecho internacional de derechos humanos, los países están en la obligación de priorizar la autonomía y la autodeterminación de las mujeres al asegurar su derecho a acceder a servicios completos de salud reproductiva (Comité de Derechos Econòmicos Sociales y Culturales. Observación Generale 14. https://www.refworld.org.es/publisher,CESCR, GENERAL,47ebcc492,0.html71, accedido el 05/Mar/2019). Como lo establece la Constitución Política de El Salvador, los tratados internacionales ratificados por el Estado son leyes en el país, y estos prevalecen en caso de conflicto con una ley nacional 4. Por tanto, como signatario de estos instrumentos internacionales y regionales, El Salvador tiene la obligación de respetar, proteger y hacer cumplir estos derechos humanos 41 .

El derecho a la salud reproductiva es un derecho reconocido por el derecho internacional de los derechos humanos 42 (Comité de Derechos Econòmicos Sociales y Culturales. Observación Generale 14. https://www.refworld.org.es/publisher,CESCR,GENERAL,,47ebcc492,0.html71, accedido el 05/ Mar/2019). Conforme a la OMS, la salud reproductiva supone el respeto, protección y garantía de la salud reproductiva en tres dimensiones: física, mental y social 43 . En esta medida, los Estados tienen una obligación de carácter positivo de asegurar el goce del derecho y un deber negativo de no privar a las personas del goce y ejercicio de su derecho fundamental 44 . 
Diversos cuerpos legales internacionales han hecho varios llamamientos a El Salvador para que modifique su legislación que prohíbe de manera absoluta el aborto. En el 2007, el Comité de Derechos Económicos, Sociales y Culturales de las Naciones Unidas (CESCR) expresó su preocupación por la discriminación que enfrentan las mujeres en El Salvador y la ilegalidad del aborto, que conlleva a los abortos clandestinos como una de las principales causas de la muerte de las mujeres. De esta medida, el CESCR recomendó al Estado reformar la ley y considerar las exenciones a su legislación 44.

Por su parte, en el 2010, el Comité de Derechos del Niño (CDN) ya había expresado su preocupación por el alto número de embarazos adolescentes y la falta de resultados de las medidas preventivas adoptadas por el Estado Parte al respecto, [así como] el hecho de que esta prohibición absoluta puede llevar a las niñas a recurrir a prácticas de aborto inseguras y clandestinas, a veces con consecuencias fatales 45. En consecuencia, el CDN recomendó a El Salvador promover y garantizar el acceso a la educación sexual y los servicios de salud reproductiva para todas las personas y solicitó considerar revisar las disposiciones del Código Penal que penaliza la interrupción del embarazo en todas las circunstancias 45 .

Posteriormente en el 2014, el CESCR nuevamente reiteró su preocupación por la continua criminalización del aborto, señalando que esta afecta desproporcionadamente a las mujeres pobres y menos educadas en particular 25, y enfatizando en la escasez e insuficiencia de los servicios de salud sexual y reproductiva, en particular para las niñas y las mujeres, que da lugar a las altas tasas de mortalidad materna y embarazo adolescente. El CESCR instó a El Salvador a revisar su prohibición total del aborto y recomendó que asegure la accesibilidad y disponibilidad de los servicios de salud sexual y reproductiva.

En marzo de 2017, el Comité para la Eliminación de Todas las Formas de Discriminación contra la Mujer (Comité CEDAW) recomendó a El Salvador modificar su legislación que penaliza el aborto y generar medidas para garantizar el acceso a los servicios de salud reproductiva 25.

Luego, en noviembre de ese mismo año, el Alto Comisionado de las Naciones Unidas para los Derechos Humanos Zeid Ra'ad Al Hussein manifestó su preocupación por que como resultado de la prohibición absoluta en El Salvador del aborto, las mujeres están siendo castigadas por abortos espontáneos y otras emergencias obstétricas, acusadas y condenadas de haberse inducido la terminación del embarazo 46. En consecuencia, hizo un llamado a El Salvador a emprender una moratoria en la aplicación del artículo 133 del Código Penal, y a revisar todos los casos donde las mujeres han sido detenidas por ofensas relacionadas con el aborto, con el objetivo de asegurar el cumplimiento con el debido proceso y estándares de juicios justos 46.

Además, en mayo de 2018 el Comité de Derechos Humanos también instó al Estado a que garantice el acceso legal, seguro y efectivo a la interrupción voluntaria del embarazo cuando la vida o la salud de la mujer o niña embarazada estén en riesgo 47, y cuando el embarazo es resultado de una violación o incesto o cuando no sea viable 47 . Además, el Comité recomendó suspender de forma inmediata la criminalización de las mujeres por el delito de aborto 47.

En octubre de 2018, el CDN reiteró sus recomendaciones anteriores, pidiéndole al Estado despenalizar el aborto y garantizar el acceso a servicios de aborto seguro y atención postaborto para niñas adolescentes, asegurándose de que sus opiniones siempre se escuchen y se les dé la debida consideración como parte del proceso de toma de decisiones 48.

Finalmente, en el contexto regional, en el 2018, la CIDH hizo un llamado al país para que revise las condenas de al menos 26 mujeres juzgadas tras haber tenido emergencias obstétricas y/o abortos, y modifique la legislación restrictiva actual.

No obstante, pese a los varios llamados internacionales, El Salvador aún mantiene su legislación restrictiva en materia de derechos reproductivos 49.

\section{Conclusión}

La pobreza y el desarrollo, sumado a los factores culturales que refuerzan los estereotipos sobre el rol reproductivo de la mujer y sus responsabilidades en la sociedad, la familia, el trabajo, su falta de empoderamiento económico y participación política, constituyen serios obstáculos para el alcance y reconocimiento de los derechos de las mujeres en El Salvador. Dicha situación de desventaja de las 
mujeres está patente en todos los niveles de la sociedad, y conlleva a un contexto de discriminación estructural y una mayor vulnerabilidad ante la violencia y la explotación, que se ve reflejado en la legislación que no reconoce la autonomía reproductiva de las mujeres.

Estos patrones legales, económicos y socioculturales reflejan un sesgo sistémico en El Salvador contra las mujeres. Desde los estereotipos de género arraigados en la sociedad, a las altas tasas de agresión sexual y violación, la falta de acceso efectivo a la atención médica para las mujeres embarazadas que sufren complicaciones obstétricas, y la criminalización de mujeres en situación de vulnerabilidad, incrementan la situación de discriminación estructural e impiden el completo ejercicio de vivir una vida libre de violencia.

Las leyes actuales que restringen los derechos reproductivos de las mujeres hacen distinciones, exclusiones y restricciones basadas en el sexo, que tienen el efecto de menoscabar o anular el reconocimiento, disfrute o ejercicio por parte de las mujeres de sus derechos humanos en violación de las obligaciones legales internacionales de El Salvador. Asimismo, no prevenir la coacción con respecto a la reproducción, y no garantizar que las mujeres puedan acceder a abortos legales y seguros constituye violencia de género, ya que inhibe seriamente su capacidad para las mujeres para disfrutar de sus derechos y libertades.

Así, en lugar de promover los derechos de las mujeres, la prohibición total del aborto en El Salvador exacerba y afianza la discriminación en su contra, lo que constituye una grave y sistemática violación de derechos humanos.

\section{Colaboradores}

J. S. Rodríguez Alarcón y M. F. Perico realizaron contribuciones sustanciales a la concepción o diseño del trabajo; contribuyeron a la redacción del trabajo, aprobación final de la versión para su publicación y están de acuerdo de responsabilidad sobre todos los aspectos del trabajo para garantizar todas las cuestiones relacionadas con la exactitud o integridad del cualquier parte del mismo.

\section{Informaciones adicionales}

ORCID: J. Sebastián Rodríguez Alarcón (00000001-8816-7611); Maria Fernanda Perico (00000002-4501-2414).

\section{Agradecimientos}

Agradecemos al Center for Reproductive Rights, Global Health Strategies y a la Fundação Oswaldo Cruz (Fiocruz) su apoyo durante la investigación y redacción de este artículo.

\section{Referencias}

1. Código Penal de El Salvador de 1998. Decreto n. 1030. Diario Oficial 1997; 10 jun.

2. Código Penal de El Salvador de 1973. Decreto n. 270. Diario Oficial 1973; 13 feb.

3. Código de Salud de El Salvador de 1988. Decreto n. 955. Diario Oficial 1988; 5 nov.

4. Constitución de la República de El Salvador. Decreto n. 38. El Salvador reconoce a la persona humana como el origen y el fin de la actividad del Estado, que está organizado para la consecución de la justicia, de la seguridad jurídica y del bien común. Asimismo reconoce como persona humana a todo ser humano desde el instante de la concepción. Diario Oficial 1983; 16 dic.

5. Center for Reproductive Rights, Excluidas, Perseguidas, Encarceladas. El Impacto de la criminalización absoluta del aborto en El Salvador. https://www.reproductiverights.org/sites/crr. civicactions.net/files/documents/crr_ElSal vadorReport_Sept_25_sp.pdf (accedido el 04/ Mar/2019).

6. Center for Reproductive Law and Policy, Persecuted. Political process and abortion legislation in El Salvador: a human rights analysis; 2001. https://www.reproductiverights.org/ document/persecuted-political-process-andabortion-legislation-in-el-salvador-a-humanrights-analysi (accedido el 04/Mar/2019).

7. Women's Global Network for Reproductive Rights. WGNRR Demands the Protection of Sexual and Reproductive Rights Defenders in El Salvador. http://wgnrr.org/wgnrr-demandsthe-protection-of-sexual-and-reproductiverights-defenders-in-el-salvador/ (accedido el 04/Mar/2019). 
8. Banco Mundial. El Salvador: contexto. https:// www.worldbank.org/en/country/elsalvador/ overview (accedido el 04/Mar/2019).

9. División de Población, Naciones Unidas. Perspectivas de la urbanización mundial: El Salvador; 2016. https://elsalvador.unfpa. org/?_ga=2.1960888.842722015.1579711179996364457.1579711179 (accedido el 04/ Mar/2019).

10. Banco Mundial. Grupo de trabajo sobre pobreza mundial: El Salvador. Washington DC: Banco Mundial; 2017.

11. Akyüz A, Yavan T, Sahiner G, Kilic A. Domestic violence and woman's reproductive health: a review of the literature. Aggress Violent Behav 2012; 17:514-8.

12. Comisión Económica para América Latina y el Caribe. La evolución de la pobreza infantil en América Latina y el Caribe; 2011 https:/www. cepal.org/sites/default/files/pr/files/51779Hojainformativa-ESP-final.pdf (accedido el 04/ Mar/2019).

13. Amnistía Internacional. $\mathrm{Al}$ borde de la muerte: violencia contra las mujeres y prohibición del aborto en El Salvador. https://www. amnesty.org/download/Documents/4000/ amr290032014es.pdf (accedido el 04/ Mar/2019).

14. Instituto Universitario de Opinión Pública. Evaluación del país a finales del 2014. Series de Informes. San Salvador: Universidad Centro Americana José Simeón Cañas; 2014

15. Organización Mundial de la Salud. Alianza mundial en pro del personal sanitario, Estudio de caso por país: Anexo 11. https://www.who. int/workforcealliance/knowledge/resources/ MLHWCountryCaseStudies_annex11_EISalvador.pdf (accedido el 14/Mar/2019).

16. Centers for Disease Control and Prevention. Healthcare access and conditions in Guatemala, Honduras, and El Salvador; 2017. https://www. cdc.gov/immigrantrefugeehealth/profiles/cen tral-american/healthcare-diet/index.html (accedido el 04/Mar/2019).

17. Naciones Unidas. Asamblea general. Informe de la relatora especial sobre la violencia contra la mujer, sus causas y consecuencias. s.l.: Naciones Unidas; 2014. (U.N. Doc. A/HRC/17/26/ Add.2).

18. Center for Reproductive Rights; Harvard T.H. Chan, Escuela de Salud Pública; Facultad de Derecho de Yale; Escuela de Salud Pública de Yale; Asociación para la Justicia Global en Salud. Voces ignoradas: experiencias de mujeres con el virus del zika. https://www.reproducti verights.org/sites/crr.civicactions.net/files/do cuments/GLP-LAC-Zika-ElSalvador-Spanishweb2.pdf (accedido el 04/Mar/2019).

19. Organización Mundial de la Salud. Aborto sin riesgos: guía técnica y de políticas para sistemas de salud. 2a Ed. Montevideo: Organización Mundial de la Salud; https://www.who.int/ reproductivehealth/publications/unsafe_abortion/9789241548434/es/ (accedido el 04/ Mar/2019).
20. Organización Mundial de la Salud. Prevención del aborto peligroso. https://www.who.int/es/ news-room/fact-sheets/detail/preventing-un safe-abortion (accedido el 19/Feb/2018).

21. Ganatra B, Gerdts C, Rossier C, Johnson BR, Tunçalp Ö, Assifi A, et al. Global, regional, and subregional classification of abortions by safety, 2010-14: estimates from a Bayesian hierarchical model. Lancet 2017; 390:2372-81.

22. Fondo de Población de las Naciones Unides. Diagnóstico de situación del embarazo en la adolescencia en la subregión andina. 1a Ed. Lima: Organismo Andino de Salud, Convenio Hipólito Unanue; 2018.

23. Human Rights Watch, International Human Rights Law and Abortion in Latin America. Human Rights and abortion; 2005. https://www. hrw.org/legacy/backgrounder/wrd/wrd0106/ wrd0106.pdf (accedido el 04/Mar/2019).

24. Fondo de Población de las Naciones Unides. Diagnóstico de situación del embarazo en la adolescencia en la subregión andina. http:// www.orasconhu.org/sites/default/files/Diag nóstico\%20de\%20Situación\%20del\%20Em barazo\%202017\%20web.pdf (accedido el 04/ Mar/2019).

25. Comité para la Eliminación de la Discriminación Contra la Mujer, Naciones Unidas. Observaciones finales sobre los informes periódicos octavo y noveno combinados de El Salvador. New York/Geneva: Naciones Unidas; 2017. (CEDAW/C/SLV/CO/8-9).

26. Federación Internacional de Planificación de la Familia. Sobreprotegidos y desatendidos: un estudio multipaís sobre las barreras legales para acceso de la gente joven a los servicios de salud sexual y reproductiva. El caso de El Salvador; 2014. https://www.ippf.org/sites/default/files/ ippf_coram_el_salvador_report_es_web.pdf (accedido el 04/Mar/2019).

27. Comisión Interamericana de Derechos $\mathrm{Hu}$ manos, Organización de Estados Americanos. Conclusiones y observaciones sobre la visita de trabajo de la CIDH a El Salvador. http://www.oas.org/es/cidh/prensa/ Comunicados/2018/011A.asp (accedido el 04/ Mar/2019).

28. Comisión Interamericana de Derechos $\mathrm{Hu}$ manos, Organización de Estados Americanos. CIDH urge a El Salvador a terminar con la criminalización total del aborto. http://www.oas. org/es/cidh/prensa/comunicados/2018/042. asp (accedido el 04/Mar/2019).

29. Cabrera O, Reingold R. De la teoría a la práctica la aplicabilidad del principio de no regresividad para proteger avances en la liberalización del aborto. In: Bergallo P, Jaramillo IC, Vaggione JM, editores. El aborto en América Latina Estrategias jurídicas para luchar por su legalización y enfrentar las resistencias conservadoras. Buenos Aires: Siglo Veintiuno Editores Argentina; 2018. p. 81-110.

30. Viterna J. The left and "life": the politics of abortion in El Salvador. Politics and Gender 2012; 8:248-54. 
31. Viterna J. Análisis independiente de la discriminación sistemática de género en el proceso judicial de El Salvador contra las 17 mujeres acusadas del homicidio agravado de sus recién nacidos. http://scholar.harvard.edu/files/vi terna/files/analysis_preliminar_17_salvadore nas_espanol_0.pdf (accedido el 04/Mar/2019).

32. Organización de Estados Americanos; Comisión Interamericana de Derechos Humanos. Informe sobre acceso a servicios de salud materna desde una perspectiva de derechos humanos. https://www.oas.org/es/cidh/mujeres/docs/ pdf/SaludMaterna2010.pdf (accedido el 04/ Mar/2019)

33. Argueta MS. La penalización absoluta del aborto en El Salvador: investigación balance de cuatro experiencias mesoamericanas en torno a la despenalización del aborto. San Salvador: La Colectiva; 2001. (Informe de El Salvador).

34. Andrade L, Adilio C. El sistema penitenciario salvadoreño y sus prisiones. San Salvador: Instituto Universitario de Opinión Pública, Universidad Centroamericana; 2015.

35. Qué país tiene la tasa de presos más alta del mundo y cuál es el de América Latina. BBC 2018; 9 may. https://www.bbc.com/mundo/ noticias-internacional-44047889 (accedido el 04/Mai/2019).

36. Organización de Estados Americanos, Comisión Interamericana de Derechos Humanos. El Salvador: Privados de Libertad. 2018. https:// www.youtube.com/watch? v=kq-kmSnLTFY (accedido el 05/Mar/2019).

37. World Prison Brief; Institute for Criminal Policy Research; Birbeck University of London. Overview El Salvador; 2016. http://www.pris onstudies.org/country/el-salvador (accedido el 05/Mar/2019).

38. Unidad de Gestión Documental y Archivos, Dirección General de Centros Penales, Ministerio de Justicia y Seguridad Pública. Guía del archivo general de la Dirección General de Centros Penales. San Salvador: Ministerio de Justicia y Seguridad Pública; 2018.

39. Küppers G, Eisenbürger G. Sentenced because of Abortion: a visit in the ilopango women's prison, El Salvador. https://eu.boell.org/ en/2017/12/14/sentenced-because-abortionvisit-ilopango-womens-prison-el-salvador (accedido el 05/Mar/2019).

40. Oficina del Alto Comisionado para los Derechos Humanos. Los derechos humanos y las prisiones: manual de capacitación en derechos humanos para funcionarios de prisiones. https://www.ohchr.org/Documents/Pu blications/training11sp.pdf (accedido el 05/ Mar/2019).
41. Oficina del Alto Comisionado, Naciones Unidas para los Derechos Humanos. Defender los derechos de las mujeres es esencial en la respuesta al Zika. https://www.ohchr. org/SP/NewsEvents/Pages/DisplayNews. aspx? NewsID $=17014 \&$ LangID $=S$ (accedido el 05/Feb/2016).

42. Naciones Unidas. Declaración y plataforma de acción de Beijing. Cuarta Conferencia Mundial sobre la Mujer. Geneva: Naciones Unidas; 1995.

43. Organización Mundial de la Salud. Constitución de la Organización Mundial de la Salud. Conferencia Institucional de la Salud. Geneva: Organización Mundial de la Salud, 1946.

44. Comité de Derechos Económicos, Sociales y Culturales, Naciones Unidas. Examen de los informes presentados por los Estados Partes de conformidad con los artículos 16 y 17 del Pacto: El Salvador. New York/Geneva: United Nations; 2007.

45. Comité de Derechos del Niño, Naciones Unidas. Examen de los informes presentados por los Estados partes con arreglo al artículo 44 de la Convención. Observaciones finales: El Salvador. New York/Geneva: Naciones Unidas; 2010. (CRC/C/OPSC/SLV/CO/3-4).

46. Hussein ZRA; Alto Comisionado de las $\mathrm{Na}$ ciones Unidas para los Derechos Humanos. Declaraciones del Alto Comisionado de las Naciones Unidas para los Derechos Humanos Zeid Ra'ad Al Hussein al final de su misión en El Salvador. https://www.ohchr. org/SP/NewsEvents/Pages/DisplayNews. aspx? NewsID $=22412$ \&LangID $=S$ (accedido el 04/Mar/2019).

47. Comité de Derechos Humanos, Naciones Unidas. Pacto International de Derechos Civiles y Políticos. Observaciones finales sobre el séptimo informe periódico de El Salvador. Geneva: Naciones Unidas; 2018. (CCPR/C/SLV/7).

48. Comité de Derechos del Niño, Naciones Unidas. Observaciones finales sobre los informes periódicos quinto y sexto combinados de El Salvador. Convención sobre los Derechos del Niño. Geneva: Naciones Unidas; 2018. (N CRC/ $\mathrm{C} / \mathrm{SLV} / \mathrm{CO} / 5-6)$.

49. Organización de Estados Americanos; Comisión Interamericana de Derechos Humanos. CIDH urge a El Salvador a terminar con la criminalización total del aborto; 2018. http:// www.oas.org/es/cidh/prensa/comunica dos/2018/042.asp (accedido el 05/Mar/2019). 